\title{
A Real-time Lane Candidates Detection Method Based On Arm Embedded Platform
}

\author{
Li Song ${ }^{1, a}$, Jiang Jingping ${ }^{2, b}$ \\ 1,2 School of Electronic \& Information Engineering of Beihang University, Haidian, Beijing, China \\ a18810254958@163.com, b18810450046@163.com \\ * Li Song
}

Keywords: Automatic driving, Advanced Driver Assistance System, Lane detection, EDLines, . Vanishing point

\begin{abstract}
The real-time lane detection and tracking on mobile embedded platform is the key module of lane departure warning and lane keeping systems. In this paper, a framework for lane candidates detection based on improved EDLines and vanishing point estimation was proposed. EDLines directly detects one pixel width and smooth continuous line, which is free of parameters and post processing, research shows that it is more robust compared to canny descriptor. Robust vanishing point estimation can not only be applied for lane candidates filtering, but also can improve the stability of lane detection. Quantity evaluation on 20000 video frames achieves $95.3 \%$ accuracy for vanishing point estimate and $95.1 \%$ higher recall for lane candidates. Taking these two key points into account, a real-time lane detection method is proposed in these articles. In experiments under daytime and nighttime, highway and urban roads, the method is robust and stable, which is $500 \mathrm{fps}$ fast in current general I7 CPU platform for 720p images, and can be applied to embedded platforms.
\end{abstract}

\section{一种实时的arm嵌入式平台车道线检测框架 \\ 李松 $1, a$, 姜精萍 $2, b,{ }^{*}$ \\ 1,2 北京航空航天大学电子信息工程学院, 海淀, 北京, 中国 \\ aemail18810254958@163.com, b18810450046@163.com \\ *李松}

关键词：自动驾驶；高级辅助驾驶；车道线检测；EDLines检测；消逝点

中文摘要. 在嵌入式平台上进行实时车道线的检测与跟踪是自动驾驶和高级辅助驾驶中车道 偏离预警与车道保持功能的核心模块, 本文基于EDLines框架改进, 提出基于消逝点的车道线 候选线段检测方案。EDLines直接获得单像素宽且平滑连续的直线, 参数不需要调整, 不需要 任何后处理, 是鲁棒的直线检测框架, 而消逝点估计不仅可以用于车道线候选线段过滤, 还 可以提高车道线检测的稳定性。基于 20000 张视频帧的定量评测表明, 消逝点估计准确率为 $95.3 \%$, 车道线候选线召回率超过 $95.1 \%$ 。本文基于以上两点提出了一种实时车道线检测方法, 在各种场景下的实验表明, 具有很高的鲁棒性, 且在720p分辨率与通用I7 CPU平台下可以达 到500fps，可用于嵌入式产品开发。 


\section{1. 引言}

\section{1 简介}

在基于计算机视觉的自动驾驶和高级辅助驾驶中，车道保持与偏离预警是至关重要的一 个功能, 它可以实现安全的自适应巡航, 其中车道线检测与跟踪是核心的模块。现有的基于 特征与基于模型的车道线检测方法, 都需要对车道线候选线段进行检测。现存的研究方法, 几乎都是采用 sobel, canny等算子与霍夫变换直线检测的框架, 该框架需要较多的参数调节, 且计算代价较大, 方法相对复杂。

EDLines作为一个不需要后处理, 无调节参数的直线检测方法, 研究表明其鲁棒性强于 sobel, canny等算子。因此, 本文将基于改进的EDLines做车道线候选线段检测。

在几何透视原理下，车道线会在远端交汇成一个消逝点。鲁棒的消逝点检测方法不仅可 以提高候选车道线的过滤从而提高检测准确率, 还可以提高车道线稳定性, 因此本文将提出 一个鲁棒的消逝点检测框架。

本文的结构安排如下。第一部分对现有主流方法做简要说明; 第二部分基于改进的 EDLines提出车道线候选线段检测框架; 第三部分提出一个基于高斯距离与线段长度投票的消 逝点估计与跟踪方法; 第四部分进行定量评测与不同场景下实验验证。第五部分进行总结和 展望。结果表明本文的方法可以兼顾实时性和检测质量。

\section{2 相关工作}

车道偏离预警(Lane Departure Warning)是高级辅助驾驶(advance driver assistance system) 的重要功能, 而当前摄像头往往被放置在挡风玻璃或者后视镜上, 由行车记录仪提供图像源。 它包括两个重要的功能模块, 即车道检测与跟踪。车道检测功能按照现有的方法 [1-4], 可以 分为基于特征的方法 [5-16]与基于模型的方法[17-21]。基于特征的方法, 利用图像的底层特征, 如边缘信息, 往往采用边缘检测的方法, 进行候选线的获取。这一类方法比较简单, 但对于 噪声, 阴影, 缺失车道线等比较敏感。基于模型的方法, 通过直线或曲线拟合的方式, 估计 几何参数, 对噪声和缺失车道线信息更为鲁棒, 但是往往计算代价更大且召回率更低。

按照边缘提取的方法, 现有的许多方法采用canny/Hough estimation of vanishing points框 架, 简称CHEVP方案 [5-9]。文[5]在将图像进行(Inverse Perspective Mapping)IPM变换后, 再经 过二维高斯滤波器, 阈值化后得到二值候选边缘。然后采用霍夫变换来统计线段, 采用 ransac 方法来过滤, 从而获得候选的车道线。该方法的主要缺陷是没有采用跟踪, 因此精度不够高, 同时在没有车道线以及有较多干扰的时候，不够鲁棒。与文[5]类似，文[6]首先采用 canny检 测边缘, 再使用霍夫变换检测直线, 使用 ransac来过滤, 由于使用了跟踪, 因此结果比较平滑 稳定。文[7-9]则采用 sobel算子来提取边缘, 然后采用与文[5-6]类似的线段检测框架。文[10] 则利用车道线的变换是暗-明-暗的灰度分布特点, 采用了条形滤波器来检测候选边缘。由于 基于边缘算子的方法对噪声比较敏感, 往往需要滤除噪声, 在文[11]中详细地比较了多种滤 波方法的效果。文[12]不仅采用了混合中值滤波先去除噪声, 也采用了改进的Hough变换, 使 得结果对噪声有较好的鲁棒性。为了剔除不相关线的干扰, 文[13]采用了梯度极大值图与自 适应的canny算子, 文[14]则采用了梯度突变算子。在所有基于边缘算子检测的方法, 边缘无 法直接使用, 往往需要基于霍夫变换的链接操作操作 [9], 这增加了计算代价。EDDrawing[15], 是一个不同于 sobel, canny等边缘检测算子的边缘检测方法, 它可以直接得到连续的边缘, 鲁 棒性更强。EDLines[16], 是基于EDDrawing的方法, 可以从边缘中直接得到单像素宽的直线。

而基于模型的方法, 模型则有样本曲线, B-snake曲线, 贝塞尔曲线等。文[17]通过B-snake 来拟合任意曲线, 而曲线的初始估计, 则是通过提出的CHEVP框架, 即Canny/Hough Estimation of Vanishing Points)框架。与上述基于特征的方法类似, 采用canny算子检测边缘, 然后将图 像从上至下分为几个区域, 采用Hough变换估计直线。在这些成对的直线的基础上, 估计地 平线和消逝点, 同时结合检测到的边缘线, 估计出道路的中间线, 最后再反推得到两侧车道 
线。该方法对于虚线, 阴影, 光照变化, 噪声等有较强的鲁棒性, 文[18]采用gabor滤波器来 估计ROI区域内每个像素的方向, 从而投票得到消逝点, 路边缘仍然采用canny算子和hough 变换来估计。最终车道线的模型由中间线, 车道宽度, 方向和曲率等参数组成。该方法对于 树阴影和行人具有很好的鲁棒性。文[19]采用了线性抛物线模型来进行检测与分类，它基于 车道线像素的灰度值高于周围非车道线像素的事实, 通过在一个小的矩形区域内统计特征, 采用级联分类器将车道线分为 5 类。虚线, 虚实线, 实虚线, 单实线与双实线。文[20-21]则都 是采用双曲线模型来拟合求取的候选边缘点。

基于模型的方法虽然对噪声和弱的车道线鲁棒性强, 但是模型中各个场景之中不具有通 用性[3-4]，而且在迭代求取关键参数的过程中，计算代价统计较大。

为了使帧间结果更平滑稳定，需要在检测的基础上采用跟踪，主要是采用卡尔曼滤波[6] 以及粒子滤波 $[15,19]$ 的方法。现有的研究表明, 两者的选择对结果影响差异不大。

\section{EDLines检测框架}

\subsection{EDLines ${ }^{[16]}$}

EDLines是一个直线检测框架，它主要包括边缘检测和直线拟合两部分。基本步骤包括两 步:

(1) 高斯平滑去除噪声, 梯度极大值针点估计。

(2) 从某一针点开始, 在四邻域的范围内, 从针点的梯度方向开始搜寻梯度极大值点, 直到回到针点或搜寻不到梯度极大值点。针点的梯度方向为水平方向或者垂直方向。

(3) 对每一条边缘，从某一端点添加线段并重新估计线段参数，直至离线段的累计坐标 距离残差超过阈值中止，开始下一条线段。

原始的EDLines方法可以得到许多的候选线段, 其中有不少误召回会对真正的车道线造成 干扰。下面从两个方向对其进行改进。

增加基于针点的过滤。对于只包含一个针点或针点紧缩在很小的一个区域而大部分线段 并没有针点的线段, 它一定不满足车道线的条件, 因为车道线有明显的明暗纹理而使得一条 线段会存在多个针点。如果即可以减少后续的计算量，又可以减少干扰。

基于物理距离的多尺度检测。由于原处的车道线清晰度不比近处, 具体表现在越靠近车 道线消逝点, 虚车道线越短, 越难以检测到。因此, 基于该原理, 本文将图像在Y轴上分块, 从上至下等分为四块, 图像中间的两块进行上采样以增加召回率, 具体评测见4.2节。

\subsection{EDLines与Canny检测比较}

不同于sobel, canny等边缘检测算子的方法, EDdlines直接得到单像素宽, 光滑且闭合的 边缘。

图1(a)是canny检测的结果图，图1(b)是本文edline的结果。Canny检测采用matlab的edge函 数, 阈值取0.2 0.5之间选择召回率和准确率最优的结果。比较表明, canny检测的结果边缘碎 片较多, 尤其见左下侧字与车头部分, 召回率低于edlines, 不仅车头部位大部分边缘未检测 出, 且右外侧车道的车道线也没有。Edlines所得的线段更长, 且为单像素宽, 不需要后处理。 在同样的I7平台与图像尺寸下, opencv canny检测所需时间约 $3 \mathrm{~ms}$, 而edlines约 $1 \mathrm{~ms}$ 。因此, EDLines还有速度优势。

总之，采用EDlines方法有 2 个优势:

(1) 一步直接得到光滑的曲线, 不需要对 sobel, canny等算子的结果进行霍夫变换等后处 理来完全直线识别与过滤，降低了中间错误。

（2）更低的计算代价和更高的鲁棒性。由于以上两个优点，本文在原来EDLines ${ }^{[16]}$ 框架的 基础上做少许改进来进行线段检测。 


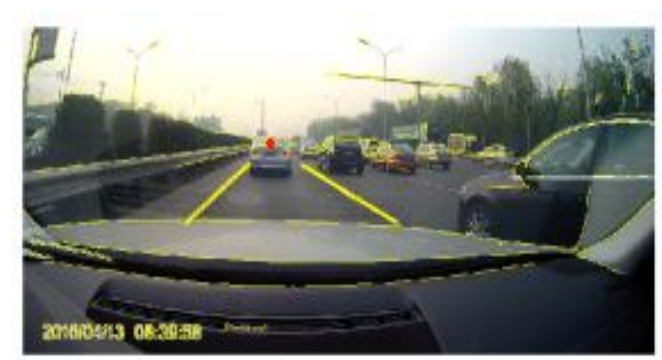

(a)

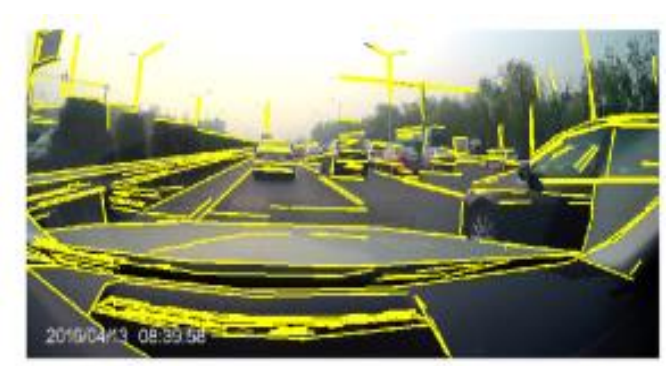

(b)

图1 (a)canny检测结果 (b)edline检测结果

\section{3. 消逝点检测跟踪与线段过滤, 车道线跟踪}

\section{1 消逝点估计与聚类跟踪}

根据透视原理, 所有车道线都会通过同一个消逝点, 本文提出使用高斯平滑的线段长度 作为权值进行投票来估计消逝点的方法。

$$
p\left(x_{0}, y_{0}\right)=\arg \max \sum \exp \left(-d\left(l_{i}, p(x, y)\right)\right) \times L_{i}
$$

如式(1), $p(x, y)$ 即像素坐标, $l_{i}$ 指线段, $L_{i}$ 指线段长度, $d\left(l_{i}, p(x, y)\right)$ 即 $\mathrm{p}$ 点到线段 $l_{i}$ 的 距离。

在每一帧获取消逝点投票结果后, 并不将其作为最终的消逝点, 而是需要对消逝点进行 跟踪平滑, 最终消逝点算法如下。

(1) 初始化: 估计出连续 $\mathrm{N}$ 帧消逝点并根据距离阈值进行聚类, 由于样本少可采用任意聚 类方法。得到最大类的样本数目 $\mathrm{n}$, 若 $n>0.6 \times N$, 则认为当前帧消逝点有效, 完成初始化, 将当前 $\mathrm{N}$ 个消逝点压入队列 $\mathrm{Heap}_{v p}$, 初始化跟踪变量 $v p_{f}$ 为 0 , 否则继续初始化。

（2）跟踪：基于前一帧有效的消逝点，取其 $\mathrm{M} \times \mathrm{M}$ 的邻域继续估计消逝点 $v p_{c}$ 。取当前栈 的最大类聚类中心 $v p_{e}$, 如果 $v p_{c}$ 与 $v p_{e}$ 距离小于 $\mathrm{TL}$, 则取 $v p_{c}$ 为当前帧消逝点结果同时将 $v p_{f}$ 置 为 0 , 否则取 $v p_{e}$ 为当前消逝点结果, 并将当前结果压入 $H e a p_{v p}$ 队首同时删除 $H e a p_{v p}$ 队尾元素, 且将跟踪变量 $v p_{f}$ 加 1 。

算法伪代码如下: 


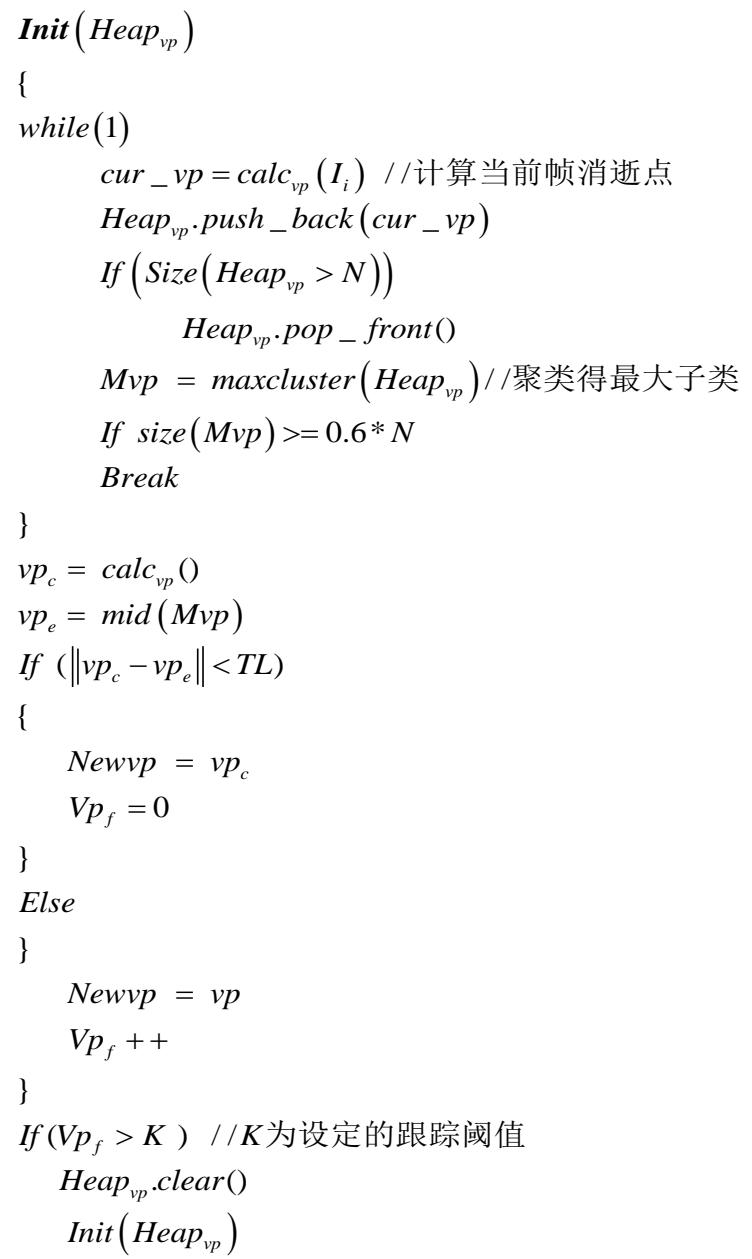

为了使结果更加平滑，可进一步采用卡尔曼滤波等方法对消逝点和检测线段进行平滑跟 踪, 由于不属于本文主要关注点, 因此下面不予详述。最终的消逝点结果见图2(b)中的红点。

本方法的消逝点估计与文 5 , 文 17 等不同在于不仅使用了高斯核对线段长度投票做了平滑, 增强了消逝点估计的稳定性。而且增加了帧间的约束, 不仅降低了计算复杂度, 且进一步提 高了消逝点的稳定性。上述参数, 本文 $\mathrm{N}=10, \mathrm{~K}=5, \mathrm{TL}=20$ 。

\section{2 线段过滤}

EDLines检测结果所得的线段虽然相比于canny, sobel等检测算子对长边缘有更高的召回, 但是仍然有较多的无效线段需要滤除。下面从两个方面进行过滤, 一是基于先验长度与角度 的过滤，一是基于消逝点的过滤。

\section{2 .1 基于几何先验知识的过滤}

车道线候选线段必须满足一定的几何关系，及长度不能太小，角度不能太小与太大。因 此该过滤规则如下

(1) 去除线段中心在图像上半部的线段, 这是车道线的位置先验。

(2) 去除长度小于 $y_{m} \times l_{t h} / h$ 的线段, $y_{m}$ 即线段中心点的 $y$ 坐标, $h$ 即图像高度，在本文 所有试验中, $720 \mathrm{p}$ 的图像该值 $l_{t h}$ 取 15 , 以像素为单位。该条件自适应地根据实际目标的远近 进行过滤, 一定程度上即放松了对原处短车道线的过滤, 又增强了图像底部干扰线段的过滤。

(3) 设定角度阈值 $a n g l e_{\text {max }}$, angle $e_{\text {min }}$ 。因为在行车过程中, 车道线与图像 $y$ 轴的绝对夹角, 不可能超过 90 度, 不可能小于 0 度。在经过大量场景的统计下, 本文选定了最佳阈值 angle $_{\max }=80$, $^{\text {angle }_{\min }}=10$ 。

该过滤的结果如图2(a), 对比图1(b)可知，绝大部分的干扰线被滤除，而有效的车道线线 段都被保留。 


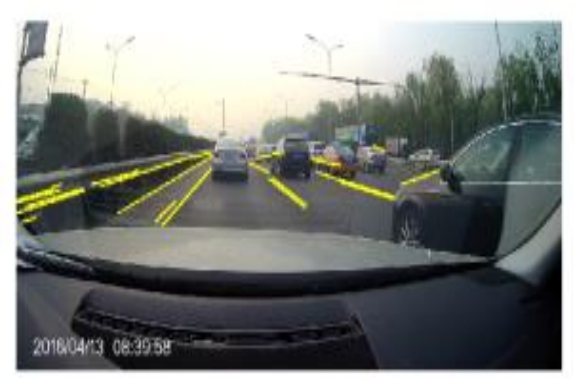

(a)

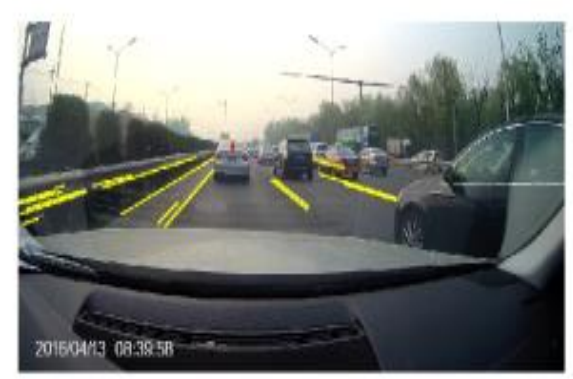

(0)

图2 (a)预过滤结果 (b)基于消逝点的过滤结果

\section{2 .2 基于消逝点的过滤}

由于所有的车道线都会交于消逝点，因此可以利用消逝点对其进行过滤，方法为计算消 逝点与相应线段中心的连线与线段的夹角 $\theta$, 将大于 $\theta_{t h}$ 的线段过滤掉, 见图 3 , 其中 $\mathrm{V}$ 为消逝 点, $\mathrm{p}$ 为线段的中点， $\theta$ 即为消逝点为线段的角度。

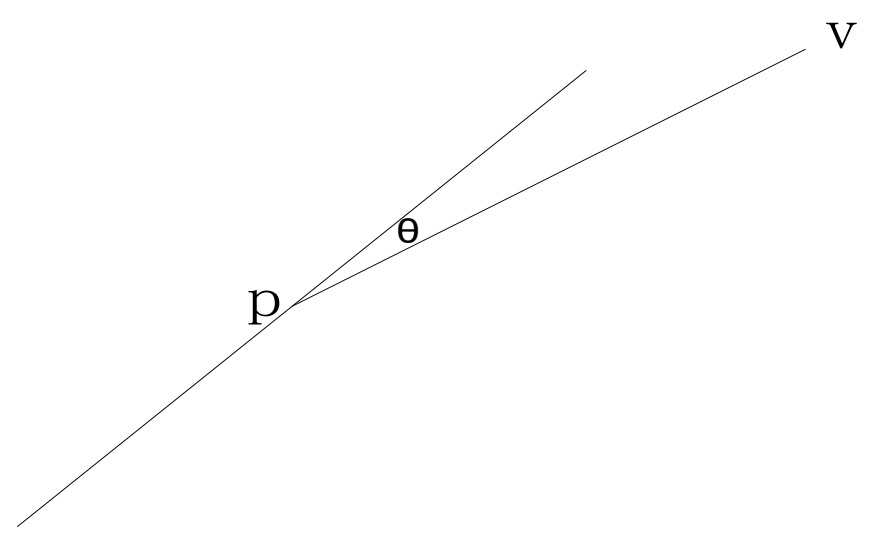

图3 基于消逝点的过滤原理, $\mathrm{p}$ 为线段中点

图2(b)为经过消逝点过滤后的候选线段，对比 $2(a)$ 可知，与消逝点夹角较大的线段都被滤 除, 剩下所有的线都是通过消逝点附近的线。最后通过合并线段和线段评分篮选后, 最终得 到车道线检测结果如下。

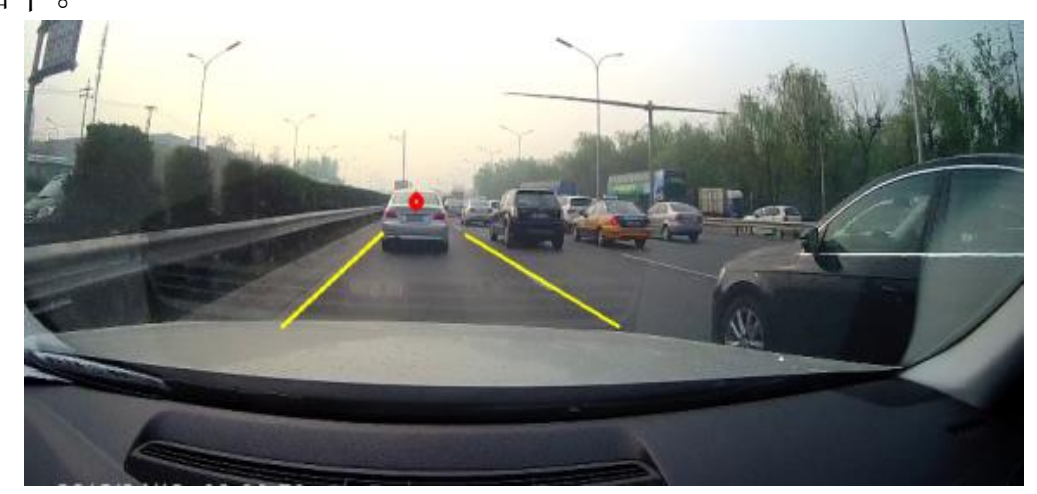

图4 消逝点与车道线检测结果

具体从图2(b)到得到图4的结果，已经超出了本论文的说明范畴，因此不做详细论述，基 本原理是依靠灰度值与长度作为线段评分标准, 同时加以两侧车道线的先验约束与帧间约束。 


\section{3 车道线跟踪}

为了进一步降低计算量, 在已经有了初始的车道线模型, 可对车道线进行跟踪, 自适应 限定ROI区域, 以进一步压缩计算量。

具体方法如下

(1) 获取当前左右车道线, 以消失点的坐标 $y_{0}$ 和车头水平线的坐标 $y_{1}$ 分别作为 $y$ 的上下 界, 延长两条车道线以获取两条车道线分别 4 个端点, 获取围住 4 个端点的最小矩形。

(2) 对于消逝点, 按照 3.1 节中的方法进行跟踪。对于车道线, 使用在该ROI区域内进行检 测的方法, 代替在全图中进行检测。该方案的优势在于有更少的干扰线段, 以及更低的计算 代价，通常ROI区域为原图尺寸的1/9以下，因此计算效率提高9倍。

(3) 更新消失点位置和ROI区域, 进行新一轮的检测。

\section{4. 实验分析与比较}

\section{1 数据库}

目前对车道线检测的公开评估数据库只有Caletch Lanes Datase ${ }^{[5]}$, 然而其标注数据较少, 且摄像机视角都是俯视图。而本文的算法主要是基于后装产品提出, 因此本文将自己采集数 据并进行标注。

本文以一款畅销的行车记录仪产品作为数据采集工具，共采集了超过100个小时，包括白 天黑夜, 高速城区以及市内的视频, 通过采样标注了 20000 个视频, 原始分辨率 $1920 \times 1080$, 检测分辨率则为 $720 \mathrm{p}$ 。标注结果包括以分段直线的形式对所有车道进行标注, 同时每帧标注 了唯一一个消逝点。

\section{2 消逝点与线段检测定量评估}

消逝点与线段检测的定量评估结果如表 1 , 只评估当前车道的两条车道线, 而不包括相邻 车道, 评估结果取两车道线的均值。消逝点与线段的准确性, 即是否与真值匹配的判断准则 如下:

$$
\begin{gathered}
\left\|v p_{e}-v p_{g t}\right\|<T H_{v p} \\
\arg \min \left\|\theta_{e}-\theta_{g t}\right\|<T H_{\theta} \quad \& \& \arg \min \| d\left(l_{e}, p\left(x_{m}, y_{m}\right)-d\left(1_{\mathrm{g} t}, p\left(x_{m}, y_{m}\right) \|<T H_{d}\right.\right.
\end{gathered}
$$

其中 $v p_{e}, v p_{g t}$ 分别为估计的消逝点与消逝点真值, $\left\|v p_{e}-v p_{g t}\right\|$ 为两者欧式距离。 $\theta_{e}, \theta_{g t}$ 为检测的线段角度与车道线真值角度, $p\left(x_{m}, y_{m}\right)$ 为图像中心点, $l_{\mathrm{g} t}, i$ 为第条 $i$ 车道线真值, $l_{e}$ 为估计的线段。因此上式(3)即表示估计的线段与某最近的真值车道线是否同时满足角度约束 和距离约束。如果同时满足, 则认为该线段检测正确。本文 $T H_{v p}=40, T H_{\theta}=5^{\circ}, T H_{d}=40$, 评测时图像尺度为 $1920 \times 1080$, 为视频的原始尺度。

表 1 消逝点与线段检测评估

\begin{tabular}{|c|l|c|}
\hline 测度 & \multicolumn{1}{|c|}{ recall } & accuracy \\
\hline 消逝点 & - & 0.953 \\
\hline EDlines & 0.951 & 0.954 \\
\hline M-EDlines & 0.963 & 0.949 \\
\hline
\end{tabular}

从表1的结果看，消逝点的准确率大于 $93 \%$, 可以验证了消逝点算法的有效性。消逝点的 估计错误, 主要是车道线线段未召回导致干扰线段投票错误造成。而EDLines的召回率和准确 率均大于 $95 \%$, 由此验证了EDlines的高召回率, 同时EDLines线段检测框架是基于梯度原理, 因此召回的候选车道线都是紧邻真实车道线边缘, 由此可去除了基于聚类的方法与基于霍夫 
变换的方法需要的后处理步骤。M-EDLines, 即本文提出的多尺度EDLines, 相比于原始的 EDLines进一步提高了线段召回率, 而增加的计算代价低于 $\mathrm{O}(\mathrm{N})$, 因此, 是有效的改进。

\section{3 车道线定量评估}

在线段检测与过滤后，可以得到候选车道线线段。经过一系列的规则过滤与跟踪，最终 可得到车道线检测结果。由于超过本文内容, 所以算法不予详述, 评估定量结果如下:

表2 车道线检测结果评估

\begin{tabular}{|c|c|c|}
\hline 测度 & recall & accuracy \\
\hline 车道线 & 0.913 & 0.920 \\
\hline
\end{tabular}

在线段检测与过滤后, 从上表 2 可以看出, 车道线的召回率和准确率均低于线段检测。这 是因为, 在很多场景中, 虽然线段检测成功, 但是由于消逝点估计出错或者非车道线线段干 扰，导致车道线检测不出来或检测到错误的线段。

下面选取了白天与夜间的市内较难的场景挑选了若干关键帧样本进行显示。

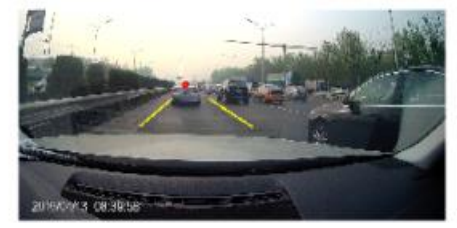

(a)

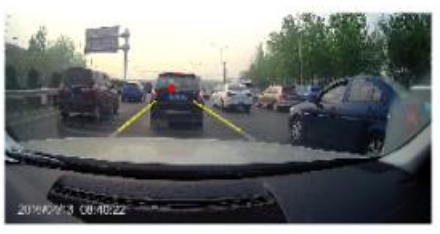

(c)

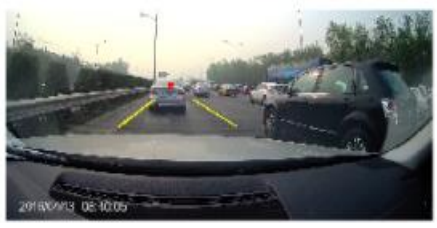

(b)

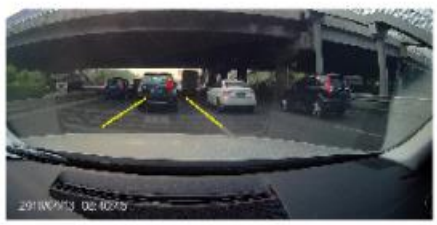

(d)

图5 白天市内场景. (a)第50帧. (b)第300帧.(c)第700帧.(d)第1400帧

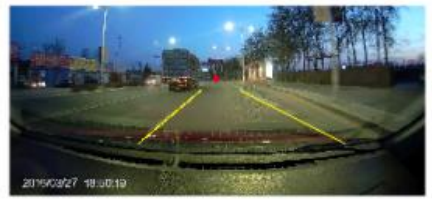

(a)

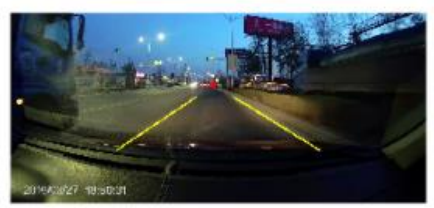

(c)

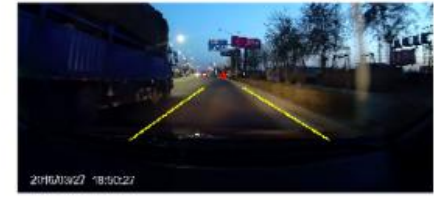

(b)

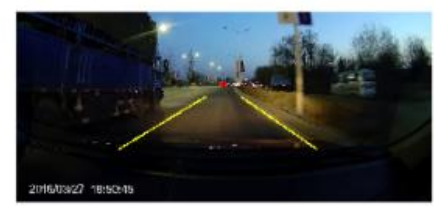

(d)

图6 晚上市内场景2 (a)第300帧 (b)第600帧 (c)第800帧 (d)第1200帧

上图5是白天在环路上的行驶视频，其车道线虽然比较模糊，但是从文章第三部分中看出 关键车道线都能够正确召回。 
图6是在最右侧车道行驶，车道线磨损非常严重且一边是路沿，不过从结果看来本文的方 法能够将其召回。

除去以上的场景, 本文也测试了雨天, 过人行道隧道等场景, 由于篇幅限制无法一一展 示。本实验的操作平台ubuntu15.04, I7-4710处理器, 未采用任何加速与并行处理方案, 用于 处理的图像大小为 $1280 \times 720 \mathrm{p}$, 实际处理ROI区域大小为 $640 \times 480$, 平均处理时间小于 $2 \mathrm{~ms}$ 。 其中EDLine线段检测的时间小于 $1 \mathrm{~ms}$ 。联发科MT6753芯片上有超过50fps的处理帧率，所以本 方案可用于实时地arm嵌入式平台车道线检测。

\section{5 结束语}

本文提出一个基于EDLines与消逝点的车道线检测框架。相比于现存的canny边缘检测与 霍夫变换, EDlines直线检测框架具有更小的计算代价和更高的召回率，同时无需参数调优。 基于本文提出的消逝点过滤方法，可以大大减少干扰线段，且利用了连续帧间信息，从而提 高车道线检测的稳定性和准确性。定量评估结果表明, 消逝点准确率超过95\%, 线段检测召 回率和准确率也都超过了 $95 \%$ 。表明了该方法用于线段检测的可行性。在各种场景下的实验 表明, 车道线的召回率也超过了 $90 \%$ 。由于计算代价小, 本文方法在不需要硬件加速算法的 情况下, 即可应用于普通的嵌入式平台。本文接下来的研究是将现有方法拓展到弯道车道线, 以及添加在更恶劣的冰雪雾皬等环境下的测试。同时, 添加换道策略, 从而实现完整的车道 保持与偏离预警模块。

\section{References}

[1] Kumar A M, Simon P. REVIEW OF LANE DETECTION AND TRACKING ALGORITHMS IN ADVANCED DRIVER ASSISTANCE SYSTEM[J]. international journal of computer science and information technologies(IJCSIT),2015,7(4).

[2] Mandlik P T, Deshmukh A B. A REVIEW ON LANE DETECTION AND TRACKING TECHNIQUES[J].

[3] Kaur G, Kumar D. Lane Detection Techniques: A Review[J]. International Journal of Computer Applications, 2015, 112(10).

[4] Hillel A B, Lerner R, Levi D, et al. Recent progress in road and lane detection: a survey[J]. Machine vision and applications, 2014, 25(3): 727-745.

[5] Aly M. Real time detection of lane markers in urban streets[C]//ntelligent Vehicles Symposium, 2008 IEEE. IEEE, 2008: 7-12.

[6] Li Y, Iqbal A, Gans N R. Multiple lane boundary detection using a combination of low-level image features[C]//17th International IEEE Conference on Intelligent Transportation Systems (ITSC). IEEE, 2014: 1682-1687.

[7] Zhang Chong,Fan Xinnan. Approach for real-time lane detection based on linear model[J]. Computer Engineering and Design,2012,01:295-299

[8] IU Xian-ru, CAI Zi-xing. Robust lane detection and tracking for the structured road[J]. Journal of Optoelectronics.Laser,2010,12:1834-1838

[9] Lin Q, Han Y, Hahn H. Real-time lane detection based on extended edge-linking algorithm[C]//Computer Research and Development, 2010 Second International Conference on. 2010: 725-730.

[10]Teng Z, Kim J H, Kang D J. Real-time Lane detection by using multiple cues[C]//Control Automation and Systems (ICCAS), 2010 International Conference on. IEEE, 2010: 2334-2337. 
[11] Srivastava S, Singal R, Lumba M. Efficient Lane Detection Algorithm using Different Filtering Techniques[J]. International Journal of Computer Applications, 2014, 88(3).

[12]S. Srivastava, M. Lumb, and R. Singal, "Improved lane detection using hybrid median filter and modified Hough Transform ," Int. J. Adv. Res. Comput. Sci. Softw. Eng., vol. 4, no. 1, pp. 30-37, Jan. 2014.

[13] Yoo H, Yang U, Sohn K. Gradient-enhancing conversion for illumination-robust lane detection[J]. IEEE Transactions on Intelligent Transportation Systems, 2013, 14(3): 1083-1094.

[14]Kim Z W. Robust lane detection and tracking in challenging scenarios[J]. IEEE Transactions on Intelligent Transportation Systems, 2008, 9(1): 16-26.

[15]Topal C, Akinlar C. Edge drawing: a combined real-time edge and segment detector[J]. Journal of Visual Communication and Image Representation, 2012, 23(6): 862-872.

[16] Akinlar C, Topal C. EDLines: A real-time line segment detector with a false detection control[J]. Pattern Recognition Letters, 2011, 32(13): 1633-1642.

[17]Wang Y, Teoh E K, Shen D. Lane detection and tracking using B-Snake[J]. Image and Vision computing, 2004, 22(4): 269-280.

[18]Zhou S, Jiang Y, Xi J, et al. A novel lane detection based on geometrical model and gabor filter[C]//Intelligent Vehicles Symposium (IV), 2010 IEEE. IEEE, 2010: 59-64.

[19]de Paula M B, Jung C R. Real-time detection and classification of road lane markings[C]//2013 XXVI Conference on Graphics, Patterns and Images. IEEE, 2013: 83-90.

[20]Wang Quan. An Improved Algorithm for Lane Detection Baesd on Hough Transformation [J]. Computer \& Digital Engineering,2014,11:2164-2167

[21]Wang Hai, Cai Fengying, Lin Guoyu, Zhang WeiGong. Lane line detection method based on orientation on variance Haar feature and hyperbolic model[J]. Journal of Traffic and Transportation Engineering, 2014(5):119-126. 